\title{
COMPENSATING POROSITY GRADIENT TO PRODUCE FLAT, MICROMACHINED POROUS
} SILICON STRUCTURES

\author{
YAMAN AFANDI ${ }^{1,2, *}$, GIACINTA PARISH $^{1 * *}$, AND ADRIAN KEATING $^{2 * * *}$ \\ ${ }^{1}$ Department of Electrical, Electronic and Computer Engineering, School of Engineering, The University of \\ Western Australia, 35 Stirling Hwy, Crawley WA 6009, Australia. \\ ${ }^{2}$ Department of Mechanical Engineering, School of Engineering, The University of Western Australia, 35 \\ Stirling Hwy, Crawley WA 6009, Australia. \\ *Corresponding author: yaman.afandi@research.uwa.edu.au \\ ** giacinta.parish@uwa.edu.au \\ *** adrian.keating@uwa.edu.au
}

\begin{abstract}
Obtaining flat micromachined porous silicon structures is extremely challenging due to the myriad of factors affecting film stress in such structures. In this work, the relationships between the current control during anodisation, average porosity, and residual stress within porous silicon thin films and micro-fabricated structures were investigated. Using a combination of electron microscopy, surface profilometry and reflectance spectroscopy, the optimum conditions to produce near zero residual stress in thin films and micro-fabricated structures were determined. The residual stress was adjusted by a continuous variation of the anodisation current in order to achieve flat structures. The flattest released porous silicon microbeams were $2.3 \mu \mathrm{m}$ thick with a peak to valley height variation of only $72 \mathrm{~nm}$ over a length of $150 \mu \mathrm{m}$. These were achieved by using an initial current density of $20 \mathrm{~mA} / \mathrm{cm}^{2}$ that was reduced down to $8 \mathrm{~mA} / \mathrm{cm}^{2}$ during anodisation. By using this method of reducing the current during anodisation, the inherent high porosity at the porous-silicon/silicon interface was reduced, which also enabled a 36\% increase in the film thickness before film delamination compared with using a constant current. These results provide a pathway to fabricate thick, optically flat micromachined multi-layer filters using a single base material (silicon) for the structural and released layers.
\end{abstract}

Keywords: porous silicon; porosity gradient; varied anodisation current; stress; microstructures

\section{INTRODUCTION}

Porous silicon (PS) has been recognized as an important material in different microelectromechanical systems (MEMS) based applications, from its use as a sacrificial layer [1] to being the core structural material for sensors [2], due to its tuneable morphological and physical properties. Porous silicon thin films are formed by a timed electrochemical dissolution of silicon in hydrofluoric acid-based solutions. The advantage of this fabrication approach is its simplicity and compatibility with microelectronic processes, with the ability to achieve different mechanical, thermal, electrical and optical properties by controlling anodisation parameters such as current density and HF concentration. However, non-uniformity in the porosity formation during anodisation can lead to internal stress, causing deformation in released MEMS-based PS devices, affecting both optical and mechanical performance [3]. Ohji et al. [4] and Lammal et al. [5] have proposed techniques to form released PS structures for accelerometers and optical filters, however, the released MEMS devices suffered from significant bending due to large porosity gradients in the films. Achieving optically flat, uniform porosity PS structures is an important criterion for high quality optical MEMS devices.

The fabrication of PS is initiated at the top surface of the Si wafer and subsequent layers are formed at the underlying PS/substrate interface. For moderately doped films, the porosity inhomogeneity is believed to be 
mainly due to depletion of the HF concentration at the PS/Si interface as the anodisation progresses [6], which results in low porosity at the top surface and higher porosity at PS/Si interface. Using constant current anodisation for thick PS films can result in significant porosity gradients, and the high porosity at the PS/Si interface can even result in unintended film detachment from the silicon substrate [7]. However, by controlled variation of current during anodisation, great uniformity of the porosity and therefore reduced stress can be achieved, allowing fabrication of released MEMS-based PS structures [8], achieving high thickness multilayered PS films [9] and improving sensing capability in Bragg reflectors [10].

The aim of this work is to alter the current during the electrochemical etch process to determine if both low in-plane stress and low residual stress can be achieved within the film. However, direct measurement of stress gradient is difficult to achieve in unreleased films. To assist this characterisation process, the residual stress of films was mapped for unreleased PS films fabricated by different current recipes, as the achieving slightly tensile residual stress results in flat to the flatness of doubly-clamped microstructures [11]. Once the residual stress was mapped, the data was used to determine the optimum conditions to achieve flat, released PS MEMS. Our investigations revealed that in addition to flat stress-free structures, an increase in the maximum thickness without delamination was observed over that which can be achieved at constant current. To understand the conditions required to produce low-stress films and flat-released microstructures in a highly stable and reproducible manner, nominally the same Si wafers were evaluated from two different vendors. These studies provide important findings to allow the transition of PS-MEMS technologies into microfabrication MEMS foundries.

\section{EXPERIMENTAL}

PS layers were fabricated by electrochemical etching in a solution of $15 \%$ hydrofluoric acid in ethanol. It is well known that the porosity depends strongly on certain fabrication parameters such as current density, with an increase in the current density causing the porosity to rise, until the critical current density for electropolishing is reached [12]. Boron-doped, 2" Si wafers (100) with thickness $275 \mu \mathrm{m} \pm 25 \mu \mathrm{m}$ were obtained from two different suppliers (WaferWorld and SiliconQuest) to study the porosity profile with depth. Both wafers have very close nominal resistivity of $0.08-0.12 \Omega . \mathrm{cm}$ for WaferWorld and 0.08-0.10 $\Omega . \mathrm{cm}$ for SiliconQuest. By considering separate vendors, the effect of minor process variations can be assessed to better understand reproducibility of the methods. However, no specific vendor is identified against the results in this report - samples are simply referred to as either from vendor \#1 or vendor \#2. The average porosity $\left(P_{a v}\right.$. $)$ was measured as a function of layer thickness (h). Constant current density samples were grown at either $10 \mathrm{~mA} / \mathrm{cm}^{2}$ or $20 \mathrm{~mA} / \mathrm{cm}^{2}$, depending on the vendor, with the current chosen to nominally achieve the same porosity of $78 \%$ at a thickness of $h=2.5 \mu \mathrm{m}$. Varied current samples were grown with conditions as described further below. The average porosity in this study was determined by fitting the measured reflectance spectrum over a wavelength range of $500 \mathrm{~nm}-900 \mathrm{~nm}$ to a Bruggeman model [13] using a genetic algorithm to extract the best-fit parameters.

The current verses average porosity was initially mapped for constant current fabrication, to determine the monotonic relationship between current and porosity. From this data, a varied current density profile $(I(t))$ was designed to compensate for the unintentional porosity gradient (or inherent) porosity gradient $(\mathrm{dP} / \mathrm{dh})_{u}$ which is known to exist for constant current [6]. The intentional porosity gradient $(d P / d h)_{i}$ is the opposing gradient that is built-in when using varied current to compensate the unintentional porosity gradient, giving an overall net porosity gradient $(d P / d h)_{n e t}$. Fig. 1 shows one profile of the current density $I(t)$ to fabricate a 2.5- $\mu$ m thick film using a current density profile with initial value $20 \mathrm{~mA} / \mathrm{cm}^{2}$ and final value $10 \mathrm{~mA} / \mathrm{cm}^{2}$. Since the total current change $(\Delta I)$ is related to the maximum porosity change and hence intentional porosity gradient $(d P / d h)_{i}$, the controllable parameter of interest is:

$$
\Delta I=I\left(t_{0}\right)-I\left(t_{\max }\right)
$$


in which $t_{0}$ and $t_{\max }$ are the initial and final anodisation times, respectively, and $I\left(t_{0}\right)=20 \mathrm{~mA} / \mathrm{cm}^{2}$ in this study. Various combinations of $I\left(t_{\max }\right)$ and $t_{\max }$ were used to produce PS layers all having the same physical thickness of $h=2.5 \mu \mathrm{m}$ but with different intentional porosity gradients as a function of depth, $(d P / d h)_{i}$, with the intention of achieving $(d P / d h)_{n e t}=0$.

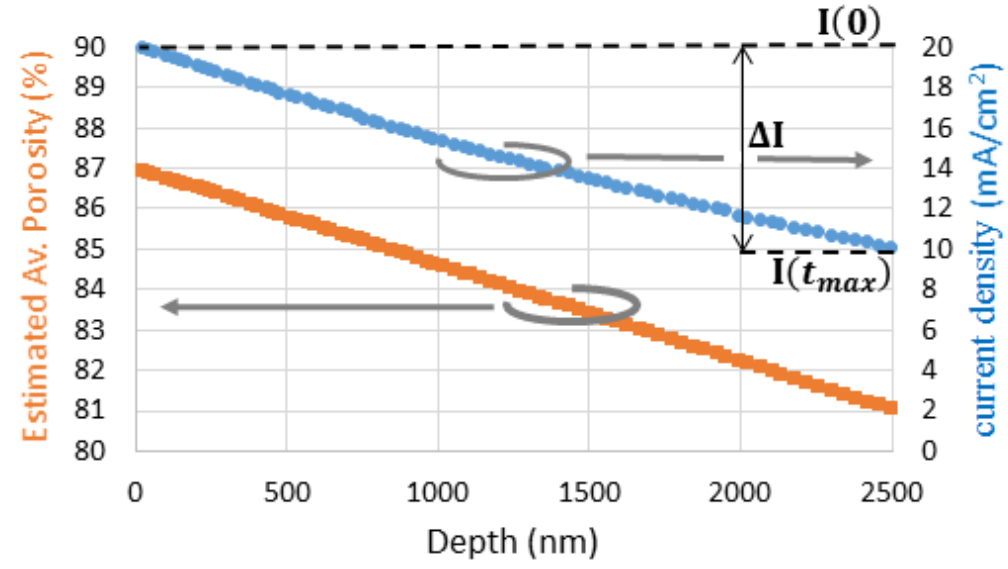

Figure 1: The left axis shows the modelled intentional porosity as a function of the depth for a 2.5- $\mu \mathrm{m}$ thick film if porosity is varying only due to current control. The right axis gives the varied current density used to create this porosity. In a real sample the current acts to compensate or enhance any non-intentional porosity gradient resulting from factors such as electrolyte depletion.

To build released MEMS structures, the films were passivated after anodisation, by annealing in a $\mathrm{N}_{2}$ atmosphere at $600{ }^{\circ} \mathrm{C}$ for $6 \mathrm{~min}$ at $1000 \mathrm{sccm}$. This process forms a $\mathrm{SiO}_{x} \mathrm{~N}_{y}$ passivation layer on the surface of pores that stabilizes PS properties and exhibits chemical resistance to allow direct photolithography processing using alkaline developer to be used on the PS film [14]. Theresidual stress $(\sigma)$ for passivated samples was obtained from film bowing measurements. In these measurements, the PS sample was cut into a rectangular shape $(\sim 3 \times 15$ $\mathrm{mm}^{2}$ ) and two location marks were made along the length of the backside (Si side) of the sample. A Zygo New view 6300 profilometer was used to measure the substrate radius of curvature $\left(\mathrm{R}_{2}\right)$ between the two marked spots to acquire the radius of curvature over a spatial range of approximately $13 \mathrm{~mm}$. Subsequently, the sample was dipped in a $10 \% \mathrm{HF}$ for $5 \mathrm{~s}$ to remove the passivation layer, then $5 \%(\mathrm{w} / \mathrm{w}) \mathrm{KOH}$ solution for approximately $10 \mathrm{~s}$ to remove the PS layer, and the radius of curvature $\left(R_{1}\right)$ was measured again, between the same marked spots. Using the Stoney's equation, the stress of each PS film was determined by:

$$
\sigma_{P S}=-\frac{E_{S i}}{6\left(1-v_{S i}\right)} \frac{t_{S i}^{2}}{t_{P S}}\left(\frac{1}{R_{1}}-\frac{1}{R_{2}}\right)
$$

where $E_{S i}$ is the bulk Young's modulus of the Si substrate, $v_{S i}$ is the Si Poisson's ratio $\left(v_{S i}=0.26\right)$, and $t_{S i}$ and $t_{P S}$ are the thickness of the Si and PS layers, which were $280 \pm 25 \mu \mathrm{m}$ and $2.5 \pm 0.2 \mu \mathrm{m}$ across all samples, respectively. By using the same marked locations for both measurements $\left(R_{1}, R_{2}\right)$, measuring over a relatively large distance $(13 \mathrm{~mm})$ and repeating each measurement twice, high accuracy was obtained from the measurement. The surface profile of released PS structures was also measured using the optical profilometer to study the flatness and measure peak to valley (PV) distance. After anodisation, released PS microstructures (beams and membrane structures) were fabricated using a process that has been reported previously [8].

SEM imaging was performed to observe the surface porosity using a Verios XHR SEM and a Zeiss SEM. Cross-section views of the PS films were taken using a FEI Helios Focus Ion Beam (FIB)/SEM, which was equipped with an array of in-lens, in-column ion beams that offers sub-nanometer milling, allowing a clear crosssection view of the porosity profile. Prior to the FIB imaging, the sample was embedded with Hard Plus Resin 
812 to eliminate damage caused by the FIB on the low-density porous films and also to improve the ion milling. Three different concentrations of resin-acetone formulation were prepared; $25 \%, 50 \%$ and $75 \%$ resin. The sample was covered with the first infiltration solution, cycled under vacuum 5 times, sonicated in a beaker for 2 minutes, and then left 24 hours in ambient atmosphere. The process was repeated twice each time using a higher concentration. After that, the sample was covered with $100 \%$ resin and left in vacuum for $24 \mathrm{~h}$. The excess resin from the sample surface was removed using a flexible plastic strip. Next, the sample was left in vacuum for another $24 \mathrm{~h}$ under $60^{\circ} \mathrm{C}$. Finally, the sample was mounted to the SEM stub using silver epoxy and then platinum coated. SEM images for the top surfaces of PS films fabricated with constant current at different thicknesses were taken to observe the porosity. These images were analysed by ImageJ software. Released porous films were analysed using SEM imaging of the top surface of the structures. Damage to some devices during fabrication allowed the morphology on the surface of the back side to be investigated by SEM.

\section{RESULTS AND DISCUSSION}

\subsection{Investigating the porosity profile in unreleased films}

Studies were initially conducted on unpassivated films attached to the substrate, with the aim of characterising films formed at constant current. Fig. 2 shows the measured unintentional average porosity $\left(P_{u}\right)$ for unreleased as-fabricated films of different thicknesses grown at constant current on wafers from the two vendors. The fitting of optical reflectance spectra in the region below $1 \mu \mathrm{m}$ failed to converge to a consistent result due to the lack of periodicity in the spectra. The extracted average porosity varied over a small range from 79-86\% for vendor \#1 and from 75-80\% for vendor \#2. Considering the slight different resistivity of both vendors, the current density was chosen to produce similar average porosity in films $2.5 \mu \mathrm{m}$ thick. For vendor \#1, a current of 10 $\mathrm{mA} / \mathrm{cm}^{2}$ produces nearly the porosity as using $20 \mathrm{~mA} / \mathrm{cm}^{2}$ for vendor \#2. Such variation is sufficient to cause relatively large variation in the target porosity and thickness as observed. As will be discussed later, this also led to different maximum thickness that could be achieved before delamination.

The average porosity initially decreases as thickness increases for films up to $1 \mu \mathrm{m}$ thick for vendor \#1, and to $1.5 \mu \mathrm{m}$ thick for vendor \#2. This indicates there may be an initially higher porosity at the top surface, with porosity then decreasing as the film moves further into the underlying substrate. As the thickness increases further, the average porosity remains approximately constant for vendor \#1 before increasing for films thicker than 3.5 $\mu \mathrm{m}$, as shown in Fig.2. For vendor \#2 the porosity increases slightly for films above $1.5 \mu \mathrm{m}$ but the films then began to delaminate for thicknesses above $3 \mu \mathrm{m}$. To try and understand the change in porosity over the range in film thicknesses for vendor \#1, SEM images were obtained for the top surface, as shown in Fig. 3. It can be seen that the pore size at the top surface then starts to widen and the pore distribution becomes more inhomogeneous for films thicker than $1.5 \mu \mathrm{m}$, which may affect both the vertical porosity profile and the average porosity. This could be due to the longer exposure to HF electrolyte during the fabrication of thicker films causing pore widening throughout. Moreover, previous studies [6] reported that the porosity increases at PS/Si interface due to the depletion of the HF. Their results combined with this study suggest that the porosity increases at both air/PS and PS/Si interfaces for thick films. At a thickness of $5.5-\mu \mathrm{m}$ using $10 \mathrm{~mA} / \mathrm{cm}^{2}$ for vendor $\# 1$ wafers and 3.3- $\mu \mathrm{m}$ using $20 \mathrm{~mA} / \mathrm{cm}^{2}$ for vendor \#2 wafers, the excessive porosity at the PS/Si interface caused the porous films to detach from the substrate. Although our results suggest that the change of porosity with depth is neither monotonic nor linear, they confirm that porosity gradient is very much a factor in film properties, and necessary to address for stable microstructure fabrication. 


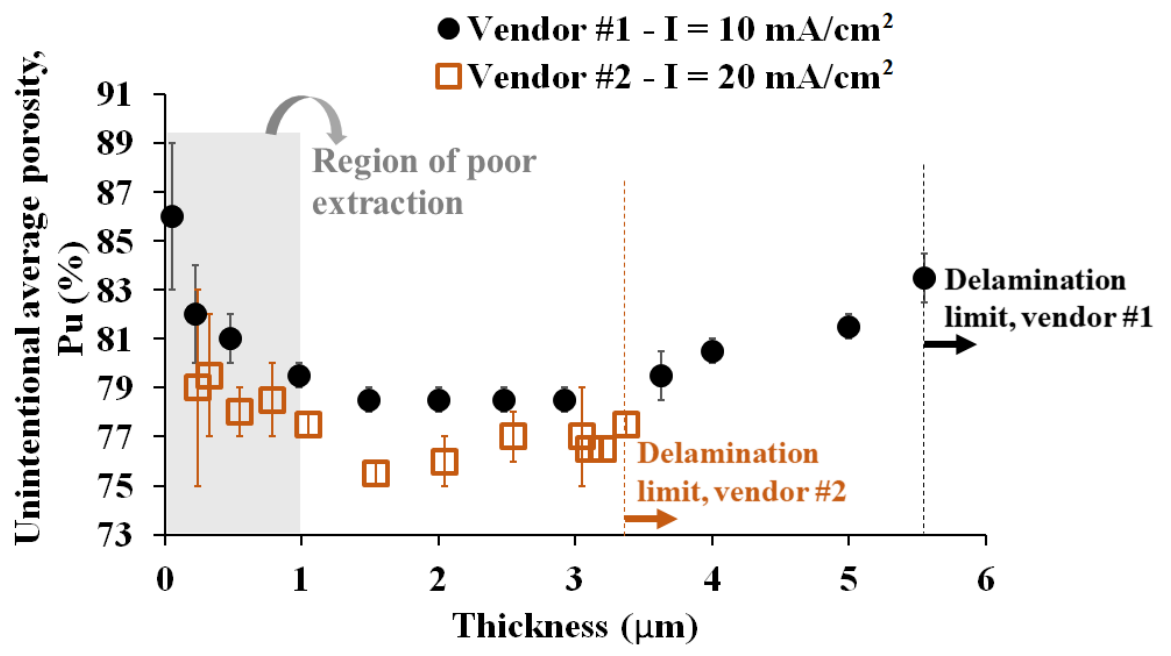

Figure 2 The measured unintentional average porosity for various porous silicon films. These porous silicon asfabricated films remained attached to the silicon substrate and were fabricated with constant current of 10 $\mathrm{mA} / \mathrm{cm}^{2}$ and $20 \mathrm{~mA} / \mathrm{cm}^{2}$ using wafers from two different vendors.
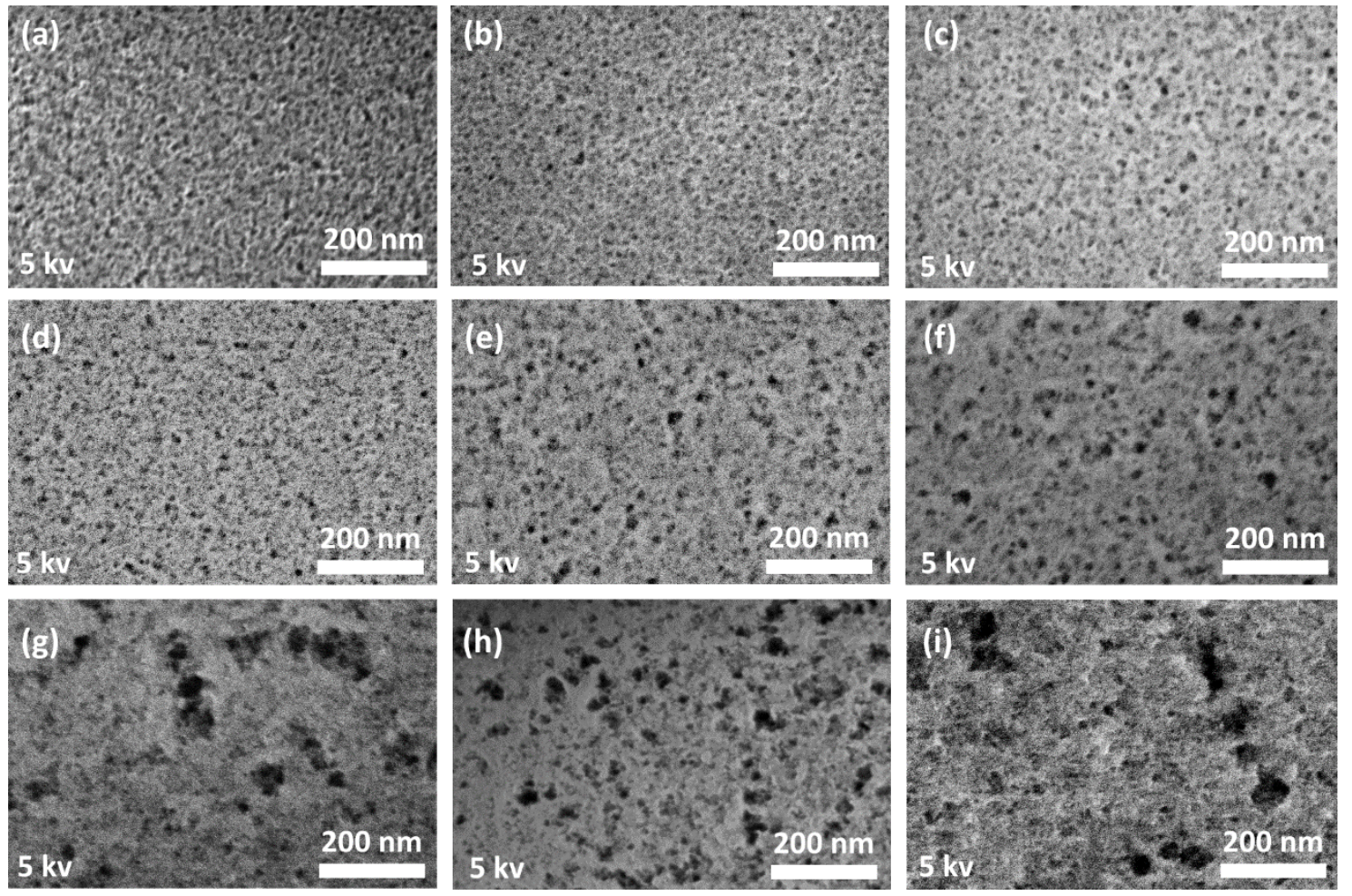

Figure 3 SEM images of the top surface of the as-fabricated (not annealed) PS fabricated from vendor \#1 wafers using a constant current of I = $10 \mathrm{~mA} / \mathrm{cm}^{2}$ with different film thicknesses; (a) $0.1 \mu \mathrm{m}$, (b) $0.25 \mu \mathrm{m}$, (c) $0.5 \mu \mathrm{m}$, (d) $1 \mu \mathrm{m}$, (e) $1.5 \mu \mathrm{m}$, (f) $2 \mu \mathrm{m}$, (g) $3.5 \mu \mathrm{m}$, (h) $4 \mu \mathrm{m}$, (i) $5.5 \mu \mathrm{m}$.

Fig. 4 shows the porosity profile in the cross-section of as-fabricated (unpassivated) thick (3.3 $\mu \mathrm{m}) \mathrm{PS}$ films which has been permeated with resin, imaged using FIB/SEM from vendor \#2. The bright region observed on the top surface area is most likely due to surface charging of the unpassivated film, and the bright region at the $\mathrm{PS} / \mathrm{Si}$ interface is most like due to trapped charges due to the high density of surface defects there. However, as a function of depth, there is significant contrast change in the SEM image which is attributed to the unintentional porosity gradient within the film. SEM cross-sectional images obtained by simply cleaving the samples did not reveal this level of detail, as the cleaving of the porous layer often led to significant damage which affected the interpretation of the data. 


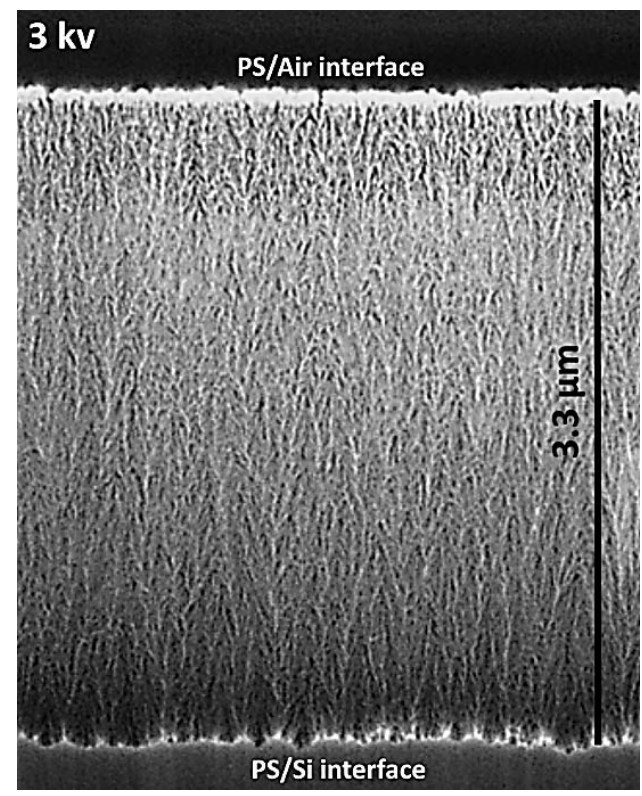

Figure 4 FIB/SEM image of a cross-section of PS film with $3.3 \mu \mathrm{m}$ thickness, fabricated using a constant current of $I=20 \mathrm{~mA} / \mathrm{cm}^{2}$.

The SEM images in Fig. 5 show the topside and the backside of a 2.5- $\mu$ m thick as-fabricated PS thin film fabricated from vendor \#1 wafers with constant current density of $\mathrm{I}=20 \mathrm{~mA} / \mathrm{cm}^{2}$ which became detached immediately after taking the sample out of the anodisation cell and drying in $\mathrm{N}_{2}$. A comparison of the SEM image of the top surface in Fig. 5a with the PS/Si interface surface in Fig. 5b shows that the pore size at the top is significantly less than at the Si/PS interface. This reinforces the premise that a large pore size at Si/PS interface caused the thicker films to detach, and indicates that using constant current is not suitable to fabricate thick MEMS-based PS structures with low stress gradient, as the porosity has a strong influence on stress [8].
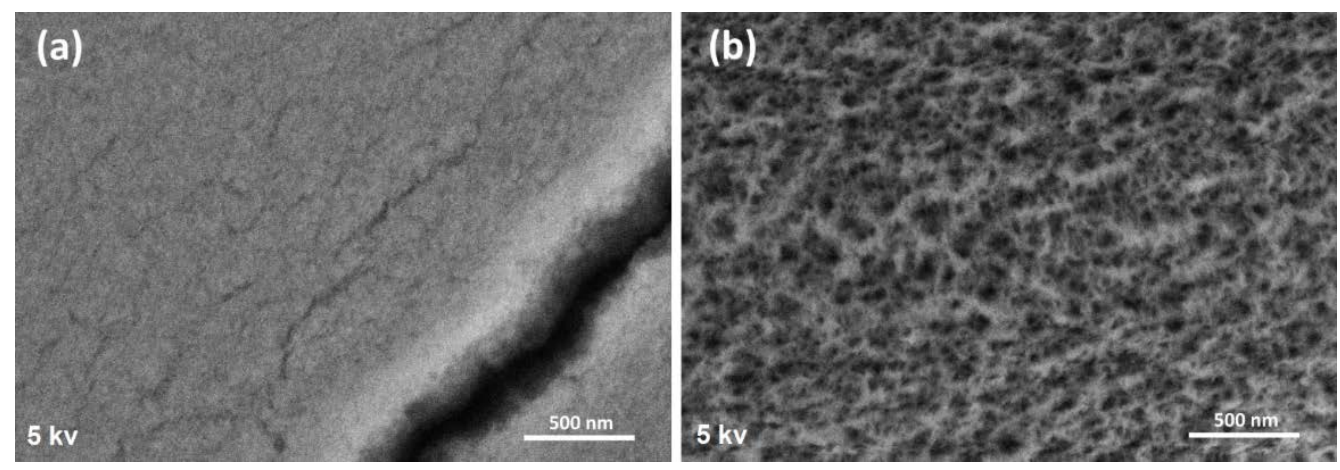

Figure 5 SEM images of a detached 2.5- $\mu \mathrm{m}$ thick as-fabricated PS film from vendor \#1 that was fabricated using constant current of $20 \mathrm{~mA} / \mathrm{cm}^{2}$, showing plan view images of (a) the top side, and (b) the bottom side.

In fact, for all films produced with constant current, no released PS MEMS could be produced, due to bending and breakage of micromachined structures. The stress gradient in the films resulted in excessive stress [8], causing cantilevers to bend considerably and microbeams to break. To achieve flat PS MEMS devices in thick films, a more uniform porosity throughout the film is required. To achieve this, we investigated current control as a method to compensate for the vertical porosity variation in the film that has been demonstrated by the investigations presented above.

3.2. Compensating porosity gradient linearly 
Following the constant current studies, $2.5 \mu \mathrm{m}$ thick films PS films were fabricated from wafers from vendor \#2 using a varying current density profile, with total change in current $\Delta I$ as described in Eq. 1 . Our goal was to linearly compensate the in-plane porosity gradient, with improvements in residual stress used to assess the effectiveness of the compensation. Given the non-uniformity with depth of the average porosity measured in Fig. 2, and the non-linear relation between applied current and the porosity at a given depth, we did not expect to achieve perfect compensation within the film. However, our desired was to use the varying current profile to achieve a first-order correction to the porosity variation in the film. Fig. 6 shows the measured post-annealing residual stress $(\sigma)$ as a function of the applied varied current density $(\Delta I)$. Considering the effect of the magnitude of the current change, Fig. 6 can be broken into two main regions; $0<\Delta I<10 \mathrm{~mA} / \mathrm{cm}^{2}$ and $10 \leq \Delta I \leq 18 \mathrm{~mA} / \mathrm{cm}^{2}$. Between $0<\Delta I<10 \mathrm{~mA} / \mathrm{cm}^{2}$, the residual stress was, on the whole, compressive (negative) but oscillated between compressive and tensile which may suggest that the stress in this range resulted from a variation in positive and negative stresses throughout the layer which was not perfectly balanced. This result indicates that the small $\Delta I$ might not be sufficient to cause a significant change in the porosity gradient for as-fabricated films compared with the inherent gradient at constant current.

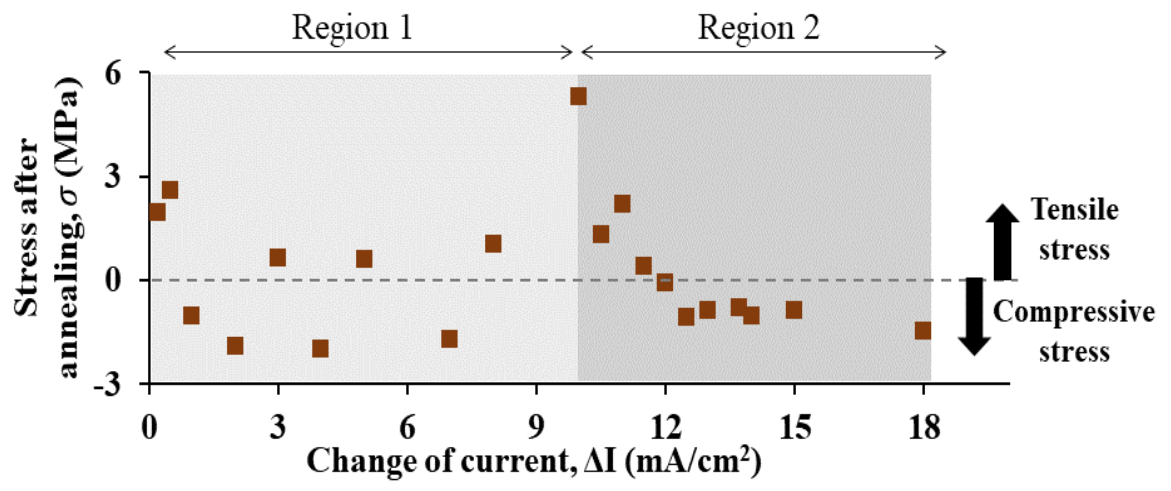

Figure 6 The effect of current change $(\Delta \mathrm{I})$ on the residual stress $(\sigma)$ for passivated films from vendor \#2. All films are $2.5-\mu \mathrm{m}$ thick.

In the range of $10<\Delta I<18 \mathrm{~mA} / \mathrm{cm}^{2}$, the residual stress decreased as shown in Fig. 6, crossing zero at $\Delta I$ $=12 \mathrm{~mA} / \mathrm{cm}^{2}$, with higher values of $\Delta I$ resulting in a residual stress that plateaued to an almost fixed compressive stress of -1 MPa. In the central regional around $\Delta I=10 \mathrm{~mA} / \mathrm{cm}^{2}$, the stress peaked at a relatively high tensile value of 5.3 MPa. Xiao et al. [11] have previously demonstrated that the stress varies correspondingly with the porosity. Karim et al. [15] have observed a complex interplay between the stresses within a relatively simple bilayer porous film, with high tensile stress reported when a thin low-porosity layer exists above a high porosity one. These observations combined with the work presented here suggest a complex interplay of stress needs to be considered when designing multilayer distributed Bragg reflector (DBR) structures made from alternating porosity layers.

After considering the residual stress results for passivated (annealed) films, five different PS samples were processed to study the flatness of released single layered microstructures: the sample with the highest residual stress at $\Delta I=10 \mathrm{~mA} / \mathrm{cm}^{2}$, two samples in the range $0<\Delta I<10 \mathrm{~mA} / \mathrm{cm}^{2}$ and two samples in the range 10 $<\Delta I<18 \mathrm{~mA} / \mathrm{cm}^{2}$, including the zero stress sample at $\Delta I=12 \mathrm{~mA} / \mathrm{cm}^{2}$. It should be noted that although the films used for microstructure fabrication were passivated after anodisation (consistent with the samples shown in Fig 6), subsequent HF immersion during electropolishing would have removed the surface passivation. The released devices had not undergone another (re-)passivation (another anneal step) and as a result, it was known that the films would possess stress shifted slightly in the compressive direction [8] compared to that shown in Fig. 6.

Fig. 7(a - e) shows SEM images of suspended doubly anchored microbeams and membrane devices from the five samples, which had varied current densities of $\Delta I=3,5,10,12,15 \mathrm{~mA} / \mathrm{cm}^{2}$. From Fig. 6, films produced 
with $\Delta I=3 \mathrm{~mA} / \mathrm{cm}^{2}$ and $5 \mathrm{~mA} / \mathrm{cm}^{2}$ showed tensile residual stress of $0.65 \mathrm{MPa}$ and $0.6 \mathrm{MPa}$, respectively. The yield at these current recipes was poor and most microbeams were fractured as illustrated in Fig. 7a and 7b. The observed bowing is a clear indication of stress gradient in these films. The poor yield is expected to be due to processing rather than excessive stress - for example the electropolishing release step involves considerable $\mathrm{H}_{2}$ emission at the Si surface which can damage the low modulus films while in the electrolyte during release. The removal of the substrate from underneath these beams means the stress can only be relieved at the anchors and internally between layers. SEM images in Fig.8 indicate a smaller pore size distribution of the top side compared to the back side of a doubly anchored microbeam fabricated using $\Delta I=5 \mathrm{~mA} / \mathrm{cm}^{2}$. As previously discussed this suggests porosity is increasing with depth, similar to that which was observed for the film using constant current shown in Fig. 5. This finding suggests that the porosity gradient at the back interface was not compensated adequately using this recipe.

(a)
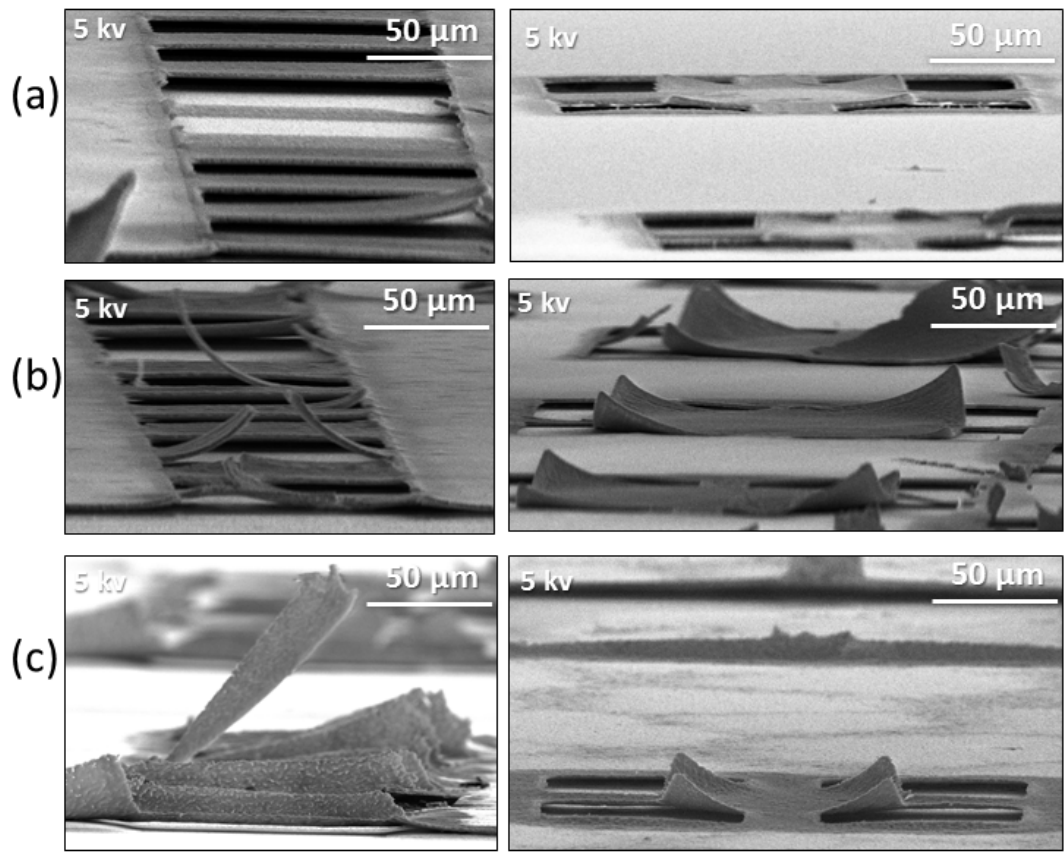

(d)
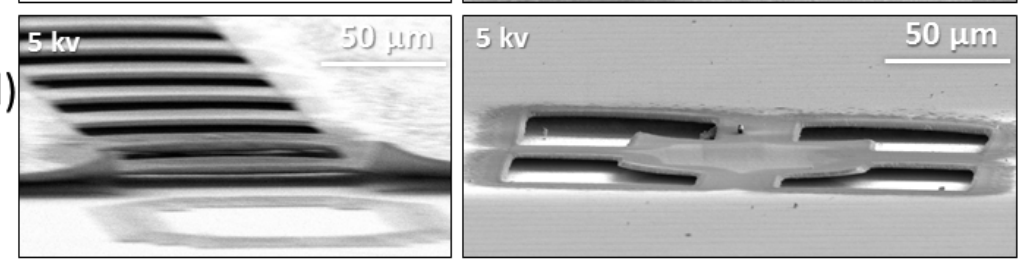

(e)
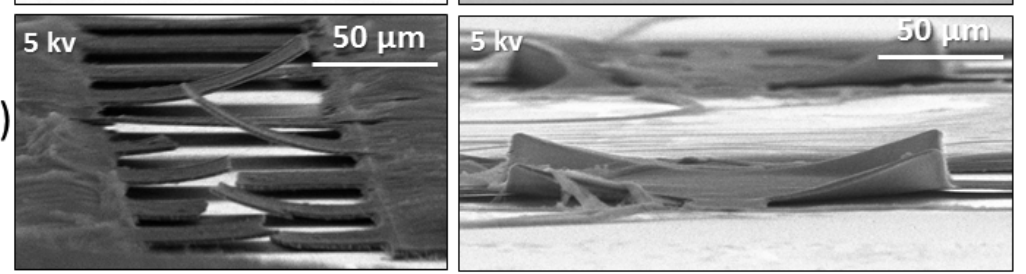

Figure 7 SEM images of suspended doubly anchored microbeam $100 \times 40 \mu \mathrm{m}^{2}$ and membrane structures using (a) $\Delta I=3 \mathrm{~mA} / \mathrm{cm}^{2}$, (b) $\Delta I=5 \mathrm{~mA} / \mathrm{cm}^{2}$, (c) $\Delta I=10 \mathrm{~mA} / \mathrm{cm}^{2}$, (d) $\Delta I=12 \mathrm{~mA} / \mathrm{cm}^{2}$ and (e) $\Delta I=15 \mathrm{~mA} / \mathrm{cm}^{2}$. These microstructures had their passivation removed during the electropolishing step and had not undergone a final

(re-) passivation anneal afterward. All samples are 2.5- $\mu$ m thick. 

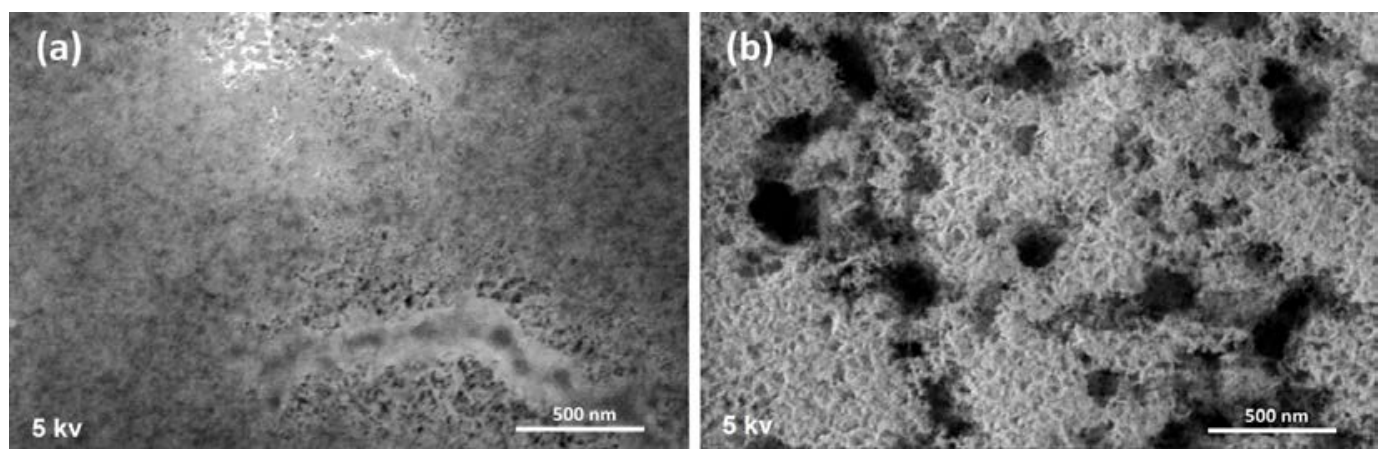

Figure 8 SEM of doubly anchored microbeam fabricated using $\Delta I=5 \mathrm{~mA} / \mathrm{cm}^{2}$ showing (a) the top side, and (b) the backside of a bent beam.

When the current was adjusted to achieve $\Delta I=10 \mathrm{~mA} / \mathrm{cm}^{2}$, the stress data in Fig. 6 suggests the highest tensile stress was obtained. This is in agreement with Fig.7c which shows significant damage, especially at the anchors, suggesting high tensile stress lead to the failures here. Interestingly, under these conditions, broken beams were deflected upwards but contained little curvature. This is indicative of anchor stress and not stress gradient in the films, consistent with the high residual stress observed. Fig. 9 shows the top side and the back side of a released doubly anchored microbeam fabricated using $\Delta I=10 \mathrm{~mA} / \mathrm{cm}^{2}$. The measurements suggest that using this recipe produces larger pores at the top surface which transitioned to smaller pores at the back side.
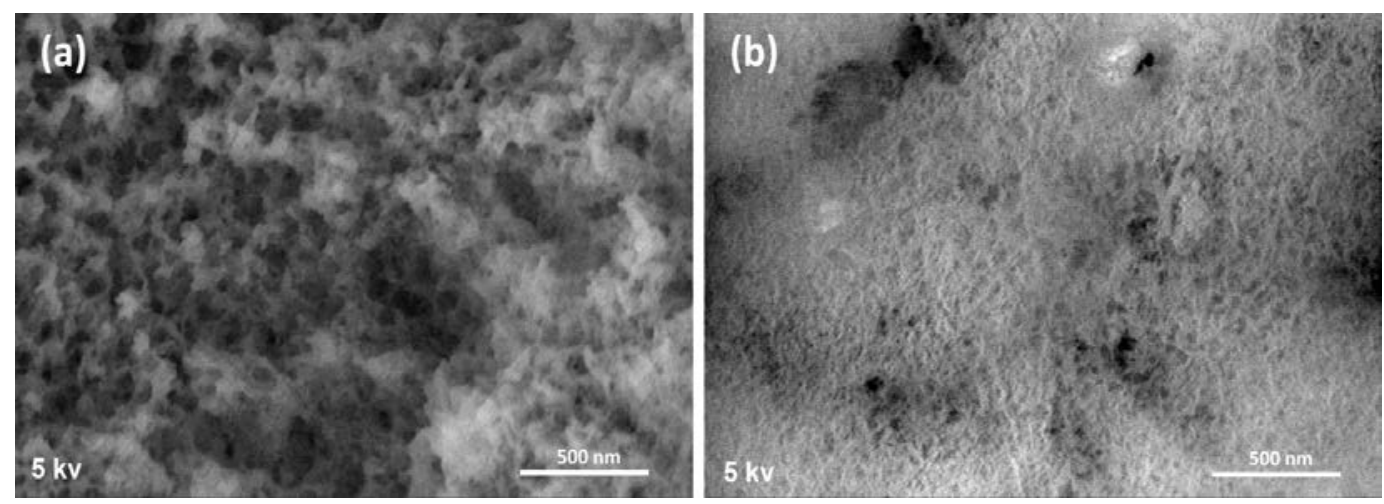

Figure 9 SEM of doubly anchored microbeam fabricated using $\Delta I=10 \mathrm{~mA} / \mathrm{cm}^{2}$ showing (a) the top side, and (b) the backside of a bent beam.

Most interestingly, at $\Delta I=12 \mathrm{~mA} / \mathrm{cm}^{2}$, the stress became almost zero as shown in Fig. 6. While near zero net-stress was also achieved from $0<\Delta I<10 \mathrm{~mA} / \mathrm{cm}^{2}$ in Fig. 6, the ability to achieve control of the stress was considerably better in the region from $10<\Delta I<18 \mathrm{~mA} / \mathrm{cm}^{2}$. This resulted in a very high yield of flat doubly clamped beams as shown in Fig. 7d. The corner bending of the PS films in this image is indicative of design stress rather than stress gradient. As with the $10 \mathrm{~mA} / \mathrm{cm}^{2}$ sample, Fig. 10 shows that under these conditions the pore sizes appear higher at the top surface than at the backside. The observed pore morphology is consistent with the back-surface result in Fig. 9b, indicating the current control in the region from $10<\Delta I<18 \mathrm{~mA} / \mathrm{cm}^{2}$ has had a significant effect on controlling porosity within the film. In the case of multilayer films, including low porosity layers can act to relax the stress from higher porosity layers as suggested by M. Karim et al. [15] and may act to increase the rigidity of the beams. Given that the porosity does not appear to be constant, despite the residual stress being near-zero, it is possible that the vertical porosity profile that results under these conditions affords such stress relief. At larger current variations of $\Delta I=15 \mathrm{~mA} / \mathrm{cm}^{2}$ and above, a compressive residual stress resulted, causing increased curvature of the plate structure shown in Fig.7e. 

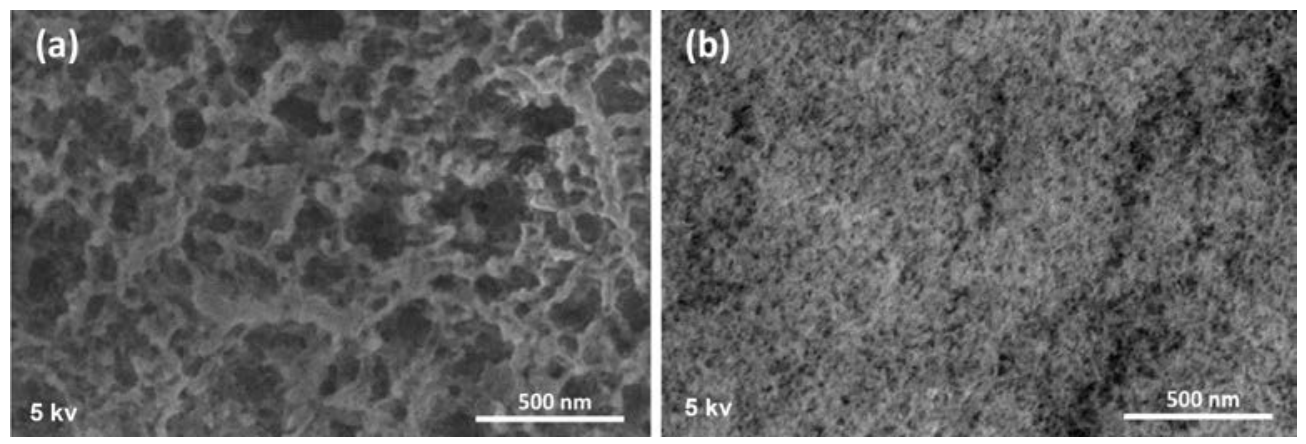

Figure 10 SEM of doubly anchored microbeam using $\Delta I=12 \mathrm{~mA} / \mathrm{cm}^{2}$ showing (a) the top side, and (b) the backside of a bent beam.

To study the flatness of these released PS-MEMS devices, the peak to valley (PV) of the surface profile of microbeams was measured. Fig. 11 shows the surface profile of these structures on the PS-MEMS devices fabricated using $\Delta I=12 \mathrm{~mA} / \mathrm{cm}^{2}$. It can be seen that the Si under the microbeams exhibited a smooth surface, indicating that the electropolishing achieved uniform etching under the released PS microstructures. The thickness of the PS structures was $2.3 \mu \mathrm{m}$ and the etched gap was $3.7 \mu \mathrm{m}$ for the microbeam and $2.5 \mu \mathrm{m}$ for the membrane. Fig.12 shows the measured PV/length of microbeams and membrane structures for 4 recipes. The measurement of the PV for structures from films fabricated using $\Delta I=10 \mathrm{~mA} / \mathrm{cm}^{2}$ could not be detected due to excessive bending in the structures. The PV/length and the roughness values $(0.48 \mathrm{~nm} / \mu \mathrm{m}$ and $7 \mathrm{~nm}$, respectively) for $\Delta I=$ $12 \mathrm{~mA} / \mathrm{cm}^{2}$ are the lowest among the recipes indicating this recipe achieved not only high yield but the best optical quality beams. The measured PV of PS in this study obtained a better flatness compared with previous studies which investigated only the effect of residua stress [8]. Our results indicate that mapping and finding conditions to simultaneously minimize the residual stress and bending is important in achieving the best results, and that addressing net porosity gradient is an important part of the conditions to be mapped.

Fig. 11b shows the surface profile of a membrane structure. This structure is a key platform structure for designing microcavity infrared optical filters. The bending at the edges of the membrane structure can be avoided by designing alternative structures where the edges are supported by the anchor [16]. Nevertheless, these preliminary results suggest suitable thickness, gap, surface, roughness and flatness that can be obtained for filters operating in the infrared range. Additionally, the recipe using current variation of $\Delta I=12 \mathrm{~mA} / \mathrm{cm}^{2}$ indicated that layers up to $4.5-\mu \mathrm{m}$ thick could be produced, 36\% thicker than achieved using a constant current density of $I=20$ $\mathrm{mA} / \mathrm{cm}^{2}$. The ability to form thick films of high porosity is important when fabricating multilayered DBR stacks which are required to support the development of high-quality infrared filters. While this work has considered only single layer films of porous silicon, the approaches taken to balance layer stresses within the entire film and consider conditions for minimizing stress and net stress gradient, will be important to achieve flat, multilayer film stacks. 
(a)

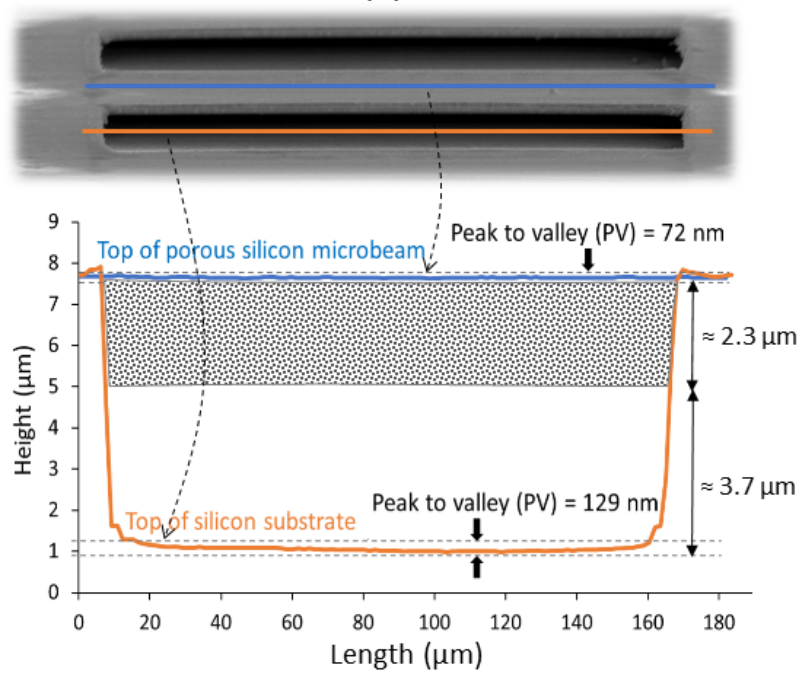

(b)

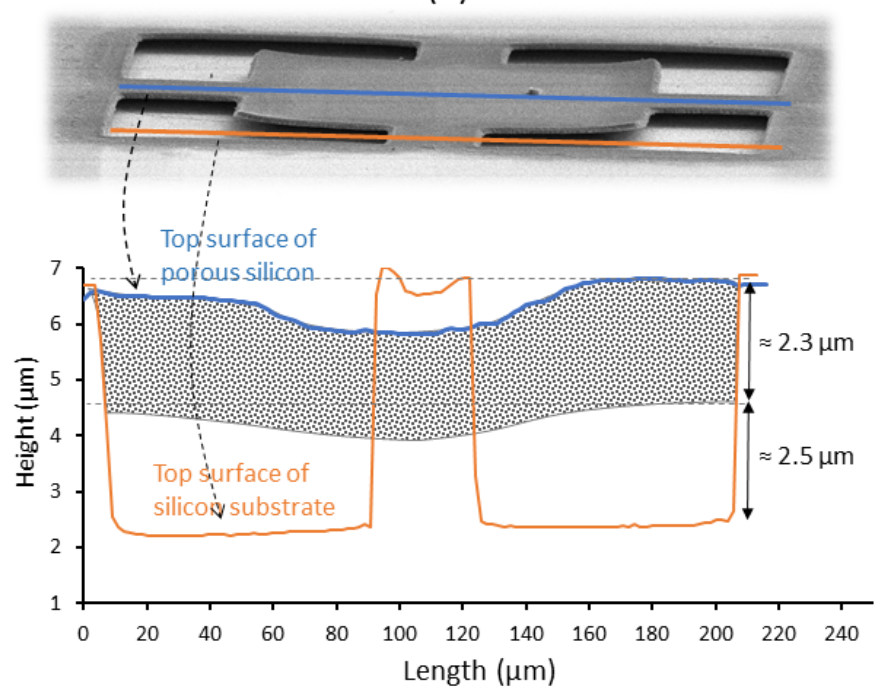

Figure 11 The surface profile of a (a) microbeam and a (b) membrane structure on a sample fabricated using $\Delta I$ $=12 \mathrm{~mA} / \mathrm{cm}^{2}$. The shaded region showing the porous silicon region has been added for illustrative purposes only and represents our average layer thickness of $2.3-\mu \mathrm{m}$.

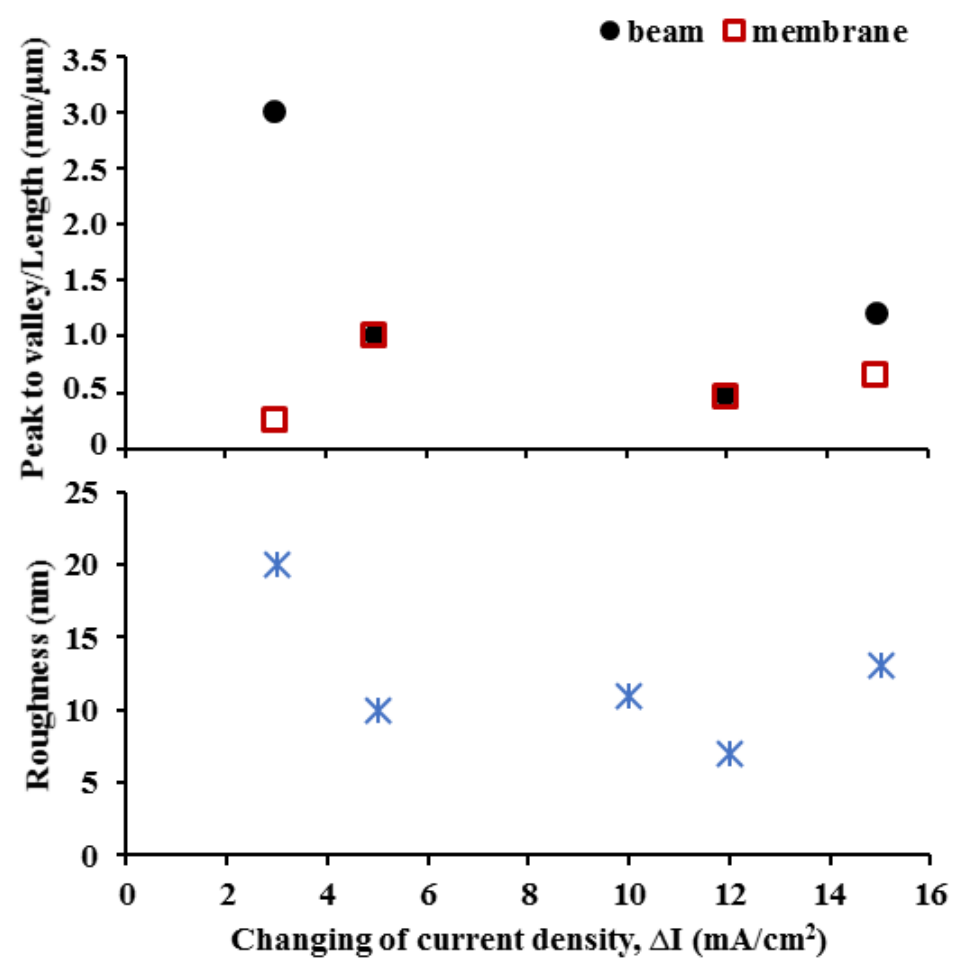

Figure 12 The peak to valley (PV) and the roughness after annealing extracted from profilometer measurements for microbeams and membrane structures using five different varied current density recipes.

\section{CONCLUSION}

The relationship between the current control during anodisation, residual stress and flatness of released microstructures was studied. Five different samples were fabricated to test the flatness and stress of porous films 
which were subsequently used as the structural layer of released MEMS structures. By changing the current from $20 \mathrm{~mA} / \mathrm{cm}^{2}$ to $8 \mathrm{~mA} / \mathrm{cm}^{2}$ during anodisation to counteract changes in porosity at the back interface, optically flat microbeams with almost zero residual stress were achieved. This recipe was also able to increase PS thickness achievable (without delamination) to $4.5 \mu \mathrm{m}$, compared to only $3.3 \mu \mathrm{m}$ when using a constant current density of $I=20 \mathrm{~mA} / \mathrm{cm}^{2}$. The study achieved excellent results in compensating the apparent stress gradient in PS films and provides an important understanding of the conditions required for fabrication of released (tunable) resonant cavity optical filters based on PS.

\section{ACKNOWLEDGEMENTS}

This work has been supported by the University of Western Australia (UWA), the Australian Research Council (DP170104266) and International Consortium of Nanotechnologies (ICON) funded by Lloyd's Register Foundation. The authors acknowledge the facilities, and the scientific and technical assistance of the Australian National Fabrication Facility at the Centre for Microscopy, Characterisation \& Analysis, The University of Western Australia, with both facilities funded by the University, State and Commonwealth Governments. The authors would also like to thank Professor Peta Clode for assistance with the resin embedded of the porous silicon film.

\section{REFERENCES}

[1] W. Lang, P. Steiner, A. Richter, K. Marusczyk, G. Weimann, H. Sandmaier, Sens. Actuators. A Phys., 43 (2010) 239-242. https://doi.org/10.1016/0924-4247(93)00655-N

[2] V. Mulloni, L. Pavesi, Appl. Phys. Lett. 76 (2000), 2523-2525. https://doi.org/10.1063/1.126396

[3] X. Sun, G. Parish, A. Keating, Sens. Actuators. A Phys. 269 (2018) 91-98. https://doi.org/10.1016/j.sna.2017.11.003

[4] H. Ohji, P.T.J. Gennissen, P.J. French, K. Tsutsumi, echnical Digest. IEEE International MEMS 99 Conference. Twelfth IEEE International Conference on Micro Electro Mechanical Systems (1999) 61-65. 10.1109/MEMSYS.1999.746753

[5] G. Lammel, S. Schweizer, S. Schiesser, P. Renaud, J. Microelectromech. Syst. 11 (2002) 815-828. 10.1109/JMEMS.2002.803278

[6] S. Bill, M. Thönissen, R. Arens-Fischer, M.G. Berger, M. Krüger, H. Lüth, Thin Solid Films, 297 (1997) 22. https://doi.org/10.1016/S0040-6090(96)09421-7

[7] N. Gaber, A. Shaarawi, D. Khalil, (2010), Advances in Optoelectronics and Micro/nano-optics (2011). 10.1109/AOM.2010.5713578

[8] X. Sun, G. Parish, A. Keating, J. Micromech. Microeng. 27(2017) 044001. https://doi.org/10.1088/13616439/aa556c

[9] J. Lascaud, T. Defforge, D. Certon, D. Valente, G. Gautier, J. Appl. Phys. 122 (2017) 214903. https://doi.org/10.1063/1.4997228

[10] R. Piya, B. Gupta, J.J. Gooding, P.J. Reece, J. Appl. Phys. 124 (2018) 163103. https://doi.org/10.1063/1.5048618

[11] X. Sun, A. Keating, G. Parish, Microporous Mesoporous Mater., 218 (2015) 88-94. https://doi.org/10.1016/j.micromeso.2015.07.010

[12] M.I.J. Beale, J.D. Benjamin, M.J. Uren, N.G. Chew, A.G. Cullis, J. Cryst. Growth. 73 (1985) 622-636. https://doi.org/10.1016/0022-0248(85)90029-6

[13] D.A.G. Bruggeman, Ann. Phys., 416 (1935) 636-664. https://doi.org/10.1002/andp.19354160705

[14] M. Lai, G. Parish, Y. Liu, J.M. Dell, A.J. Keating, J. Microelectromech. Syst. 20 (2011) 418-423. 10.1109/JMEMS.2011.2111356 
[15] M. Karim, R. Martini, H.S. Radhakrishnan, K.V. Nieuwenhuysen, V. Depauw, W. Ramadan, I. Gordon, J. Poortmans. Nanoscale Res. Lett. 9 (2014) 348. 10.1186/1556-276X-9-348

[16] N. Neumann, M. Ebermann, S. Kurth, K. Hiller, J. Micro. Nanolithogr. MEMS MOEMS 7(2008) 021004. https://doi.org/10.1117/1.2909206 